\title{
Research of the JET A-1 aircraft fuel electrification
}

\section{Igor L. Trofimov, Andrian A. lavniuk and Marharyta M. Radomska*}

Institute of Environmental Safety, National Aviation University, Kosmonavta Komarova 1, 03058 Kyiv, Ukraine

Email: troffi@ukr.net

Email: Andrian.Iavniuk@gmail.com

Email: m.m.radomskaya@gmail.com

*Corresponding author

\begin{abstract}
The paper presents the study of aviation fuel JET A-1 electrification and substantiation of the possibility to use static electricity for power generation. The modern aircraft refuelling with high-performance pumps followed by the fine filtration is a stable source of static charges generation, which raises the need to combat them. The results of the experiments show the dependence between the parameters of static charge density and the flow, flow rate, flow rate gradient and length of the pipeline. The level of the tank filling has been also accounted as a special index. We have also developed the device and the method for obtaining high voltage power, which is based on the positive use of the static electricity charges derived from the static charge converter. The further research must cover the effect of fuels electrification on the change of their operational properties, with the subsequent possibility of their modification.
\end{abstract}

Keywords: aviation fuel; electrification; dielectric liquids; electric charges; filters; pipes; charge density; device.

Reference to this paper should be made as follows: Trofimov, I.L., Iavniuk, A.A. and Radomska, M.M. (2018) 'Research of the JET A-1 aircraft fuel electrification', Int. J. Sustainable Aviation, Vol. 4, Nos. 3/4, pp.273-289.

Biographical notes: Igor L. Trofimov received his $\mathrm{PhD}$ in Technical Science in 2011 for his work on friction and wear in cars. Since 2003, he has been working as a teacher and researcher at the National Aviation University (Educational and Research Institute of Environmental Safety). He is currently an Associate Professor at the Chair of Ecology. He has participated in numerous national and international programs and projects related to the implementation of alternative energy resources in aviation. He is currently the Deputy Chairman of the aviation fuel supplying airports of Ukraine.

Andrian A. Iavniuk is going to receive his $\mathrm{PhD}$ in Biological Science for work on biological effects of long-term ionising radiation impact on common reed's seedlings. Since 2014, he has been working as an Assistant Professor at the National Aviation University (Scientific and Research Institute of Environmental Security, Department of Ecology). He has published 38 papers concerning thesis research area including five articles in specific issues, one book chapter in Springer Edition. He takes part in national and international scientific conferences as a young investigator. 
Marharyta M. Radomska received her PhD in Technical Science in 2011 for her work on the environmental safety of the fuel filling complexes. Since 2006, she has been working as a teacher and researcher at the National Aviation University (Educational and Research Institute of Environmental Safety). She is currently an Associate Professor at the Chair of Ecology. She has participated in a range of projects on the environmental impacts assessment for a variety of industries, including transport companies.

This paper is a revised and expanded version of a paper entitled 'Electrization of the RT aviation fuel as technique to generate the high voltage electric power' presented at International Symposium on Sustainable Aviation 2018, Roma, Italy, 9-11 July 2018.

\section{Introduction}

The aggravation of energy problems in the world contributes to the search for new energy sources able to provide the needs of humankind in heat and power, while ensuring high environmental safety, simplicity and safety in service. The world research community is working on the creation of hydrocarbon-based alternative fuels, as well as on the search for alternative energy sources.

It is paradoxical that some of the physical phenomena known to humankind since ancient times are currently the least studied, such as static electrification, mentioned for the first time by Thales Miletus about 2,500 years ago. In fact, the classical experiment on the electrification of amber on the wool was the beginning of the static electricity study and the same test is used in the school program to represent the phenomenon in our days. For a long time it was considered useless for application, which had distracted the attention of the scientists from it. The intensive study of electrification started at the beginning of the twentieth century, when, in connection with the rapid technological progress, the consequences of the electrostatic charges formation began to appear: explosions, fires, etc. There are numerous cases of negative manifestations of electrification in various industries: chemical, textile, pulp and paper, rubber, medicine, coal mines, transport and many others (Roizen and Medvedeva, 1995; Pribylov, 2003; Ilin and Dzhioeva, 2018). For example, according to the statistics, the damage from the static electricity only at US enterprises dealing with dust of various substances is about 100 million dollars per year (Pribylov, 2003; Taubkin, 2018). The most dangerous electrification is for the industries involving production and consumption of large quantities of flammable substances, in particular, hydrocarbon liquids. As a result, the initiators of the systematic study of the electrification of petroleum products were the largest oil companies - Shell and Esso, which had setup target laboratories to study the causes of static electricity and to improve methods for its elimination (Ilin and Dzhioeva, 2018; Taubkin, 2018).

Therefore, such a source of electric energy as static electricity can not remain unnoticed, and the problems that have arisen in the market of energy carriers in our time force to look for methods of static electricity useful application, instead of the ways of combating it. 


\subsection{Problem formulation}

The purpose of this work is to study the phenomenon of electrification of hydrocarbon liquids and to substantiate the possibility of using static electricity to generate power from the distribution of charges in dielectric liquids.

\section{Analysis of research and publications}

The use of static electricity, which arises in technological equipment due to the distribution of charges during the friction of dielectric fluid on the surface of the equipment, as a positive phenomenon is relevant and new. Modern high-performance pumps and equipment enables refuelling a large aircraft in 20-40 minutes. A mandatory element of the refuelling system is a filter of fine purification made of fibrous plastic with capacity of 3-4 thousand 1/min, high treatment efficiency and low cost. However, at the moment of refuelling of aircraft it is the filter, which leads to the intensive formation of static electricity charges (Chebotarev, 2003). The static charges have also been detected on the grid in the transfer pump of the aircraft, and they have caused burning of the filter material in the fuel filler filter. Significant manifestations of the static electricity are typical for gas stations during the fuelling of motor transport and the pumping of light petroleum products. As a result, numerous cases of self-ignition of technological equipment and cars during the filling process have been recorded (Chebotarev, 2003; Ilin and Dzhioeva, 2018; Samoilescu, 2009).

The electrification of dielectric liquids is known to be associated with the appearance of double electric layers at the surfaces of the separation of two liquid media, or at the boundaries of the separation of liquid and solid. Double electric layer is the spatial distribution of electric charges of various characters, bound to the wall of the pipe (Helmholtz layer $-10^{-6} \mathrm{~m}$ ) and the diffusion layer of ions of the opposite sign (Gouy layer) (Chebotarev, 2003). Due to the movement of the fluid along the pipe wall and the transfer of charges of the Gouy layer to the vessel, the potential difference appears between the pipe and the capacity. In case of friction of liquids on metals in the processes of flow or spray, electrification of dielectric liquids occurs due to the electrolytic separation of charges at the metal-liquid boundary. The electrification, in the case of friction of two liquid dielectrics, is caused by the existence of double electric layers on the surface of the separation of fluids with different dielectric permittivity, as a result liquid with a higher dielectric permittivity is charged positively, and the one with lower permittivity acquires negative charge (Cohen's rule) (Chebotarev, 2003). Charges of static electricity are also observed during friction of two dielectrics; semiconductors of metals of different chemical composition, or of the same composition, but of different density; metals on dielectrics; two identical dielectrics; liquid dielectrics in each other, or on the surface of solids, etc. In this case, both bodies are electrified, and their charges are the same in magnitude, but different in signs. Due to the fact that hydrocarbon fuels have low specific electric conductivity by their physical nature, they are actively electrified, and are able to store and accumulate electric charge. At a determined electrostatic charge density, the electric field strength can reach critical values, which will cause an electrical discharge. 
Among the pioneers of research in the field of the hydrocarbon fuels electrification there was the company 'Esso', which conducted tests on the model tanks of the aircraft F-100 and Vikers-Avangard (Rogers and Mundoy, 1980). In the first experiment, a large volume separator filter was used. The fuel residence time in the filter reached $107 \mathrm{sec}$, while the maximum relaxation time of the studied fuels did not exceed $1.8 \mathrm{sec}$. During the second experiment fuel with the conductivity of $4 \cdot 10^{-12} \Omega^{-1} \mathrm{~m}^{-1}$ was pumped at the rate of $\sim 1 \mathrm{~m} / \mathrm{sec}$, and the field strength in the area above the fuel did not exceed $140 \mathrm{kV} / \mathrm{m}$. Before getting into the tank, the fuel was sent through a long $(18.3 \mathrm{~m})$ rubber hose. Naturally, under such 'soft' pumping conditions the most significant consequences of electrification could not manifest. These consequences taking the form of two fires were manifested in the tests conducted by the Shell Company at the research centre in Amsterdam (Shell Research Ltd. and Shell Int. Research N.v., 1960]. The research was carried out on a rectangular tank with dimensions $1.04 \times 1.04 \times 0.69 \mathrm{~m}$. The maximum fuel flow during refuelling was $1,820 \mathrm{l} / \mathrm{min}$ and the diameter of a hose was $63 \mathrm{~mm}$. The refuelling was done through a paper filter. Fires in both cases arose after discharge from the liquid mirror in a filled tank, a few seconds after the pump was stopped. In the experiments, when a finger-shaped bar had been fixed on the roof of the tank, the sparkling began on that bar. The discharge energy was about $20 \mathrm{~mJ}$. The most thorough study of the electrification of fuels during refuelling in aircraft tanks was carried out at the Shell Research Center in Toriton (Shell Research Ltd. and Shell Int. Research N.v., 1960).

These works also confirmed the high risks levels when using clean fuels. Equipment and conditions for conducting the experiment were the same as in Amsterdam. The flow currents were detected to be up to $10 \mu \mathrm{A}$. The field strength reached the level of $800 \mathrm{kV} / \mathrm{m}$ and sometimes it was $1,250 \mathrm{kV} / \mathrm{m}$. At the last stages of filling the discharges were released: the fuels with electrical conductivity below $1 \cdot 10^{-12} \Omega-1 m^{-1}$ produced the discharges at all the studied flow rates $(1,820,1,365$ and $910 \mathrm{l} / \mathrm{min})$; the fuels with electrical conductivity $1-2 \cdot 10^{-12} \Omega^{-1} m^{-1}$ produced the discharges at the flow rates of 1,820 and $1,365 \mathrm{l} / \mathrm{min}$; and the fuel with conductivity of $\gamma=2.5 \cdot 10^{-12} \Omega^{-1} \mathrm{~m}^{-1}$ did not produce the discharges. The discharge energy was $0.5-4 \mathrm{~mJ}$.

Based on these data it was indicated that fuel with conductivity $\gamma=2.5 \cdot 10^{-12} \Omega^{-1} m^{-1}$ of was considered to be safe. This conclusion is valid, but only at the flow rates of the study.

Due to the increase in the volume of tanks of airplanes and the need to shorten their refuelling time, today such studies have become relevant again (Chebotarev, 2003; Ilin and Dzhioeva, 2018; Samoilescu, 2009). Thus, the comparison of three brands of fuels was carried out to define the threshold of sparking by the rate of pumping, at which sparking would begin in the fuelling space of the tank (Taubkin, 2018).

The obtained data indicate that the electrical properties of the studied brands of fuels are not the same. The spark rate for fuels T-7 was 700-800 $1 / \mathrm{min}$, and it was $900-1,000 \mathrm{l} / \mathrm{min}$ for TC-1 and T-1. It is noted in the study report that the estimates obtained by this method did not correlate with the existing ideas about the electrification of liquids. In particular, the fuel T-1 and TC-1, which possess different electrophysical properties, have turned to have the same threshold of sparking, while it is expected that they must be different (Taubkin, 2018). 
The most research works are currently devoted to the methods of reducing electrification. In Pinchuk (1997) works, he evaluated the efficiency of the application of the disperse capacitance at the models of the fuelling system of the aircraft on the fuelling station. Some of the research results show that the presence of a grounded rod in the pipe, located along its axis, contributes to the increase in electrification (Pinchuk, 1997; Samoilescu, 2009). The influence of the grounded grid in the pipe has also been investigated there. It has been found that the efficiency of such a grid is very small. Moreover, the presence of the grid in the pipe has increased the formation of charges by 3-10 times or more. The various types of electrostatic charge neutralisers are also studied, in particular, passive (Chebotarev, 2003) and active (Taubkin, 2018). The efficiency of passive neutralisers has been evaluated differently by various authors, while active neutralisers have the efficiency of $70 \%-80 \%$ (Taubkin, 2018). Influence of the devices design features on the electrification of fuels has been investigated as well, showing that the charge formation in the tank is the lowest if the drain pipe has a cut at the angle of $45^{\circ}$ for all investigated pump conditions (Samoilescu, 2009). As for the electrification in filters, assuming that within the nominal flow the electrification is linearly dependent on the flow rate, the absolute values of charge occurring in the filters are higher than in the pipes by an order of magnitude.

Many scientists in the world have studied the characteristics of discharges in hydrocarbon vapours, developed methods for calculating electrostatic fields, but they cover the simplest cases that coincide with the results of the experiment. In contrast, the question of the conditions for the formation and accumulation of charges is still not sufficiently studied. Attempts to substantiate theoretically the electrification of liquids contradict the experimental data. Moreover, it is not clear, where the mistake is: whether it is the basic assumptions underlying the theory, the technique of experiment, or both.

Two mechanisms are decisive when operating dielectric fluid: the formation of charge in the flow through the pipes and during filtration. Studies (Chebotarev, 2003; Samoilescu, 2009; Taubkin, 2018) have established a qualitative picture of fuel handling in these cases, on the basis of which the following conclusions could be made: the electrification of dielectric liquids is determined by three factors - the physical properties of the liquid, the structural features of the equipment and the complex of external conditions. Investigations of fluid electrification are usually carried out in complex, that is, without exclusion of any of these factors. As a result, the conclusions made based on such experiments often contradict each other. Thus, it was widely assumed that the material of the devices practically does not affect the degree of electrification, but practical results show the opposite (Scanavi, 1991).

All possible charging mechanisms can be classified into three groups (Figure 1).

Obviously, under different conditions different liquids are electrified in different ways. This suggests that such property of liquids could be determined based on relevant experimental data. But these concepts, which are the basis of the phenomenon, do not explain the fact why pure hydrocarbons are not electrified. The above examples are not a total list of all contradictions and inconsistencies, but only a brief illustration of the imperfection of our knowledge in this area. Many researchers (Rogers and Mundoy, 1980) note, that the study of the static electrification, including the one appearing in liquids, is at the stage of accumulation of experimental material. 
Figure 1 Classification of the charging mechanisms

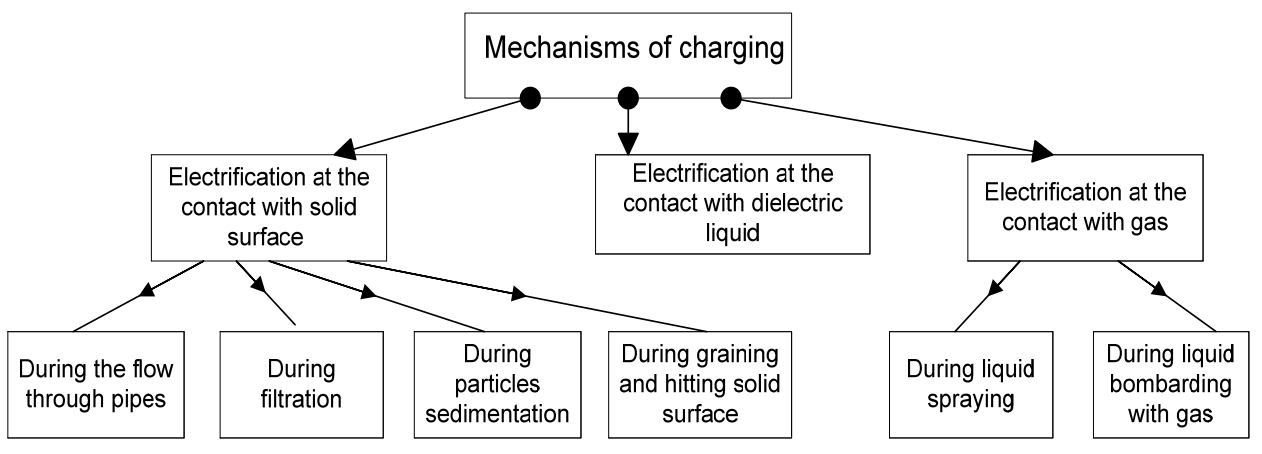

At the enterprises of aviation fuel supply, the neutralisation of charges of static electricity at filling stations during filling of the refuelled vessels is carried out by induction neutralisers of the static electricity (INSET), which are installed after the filters in the line of delivery of petroleum products. The presence of the neutraliser enables increasing the performance of the tanks filling with fuel by $70 \%$ (accounting equipment capacity). But INSET is not the only way to combat static electricity charges. In addition, in these cases, single metallisation of structures is successfully applied for equalisation of potentials or simple grounding of dangerous areas to drain charges into the ground (Roizen and Medvedeva, 1995). Taking into account the urgency of the problem of fuel protection from the accumulation of static current in aviation fuels complex additives 'Assa', 'Sigbol', which have antistatic properties, are also added. At the concentration of $0.003 \%$, they increase the electrical conductivity of petroleum products and reduce electrification.

However, the mentioned electrostatic charge mitigation measures are not very efficient. Thus, antistatic additives only reduce the electrification of petroleum products, increasing their electrical conductivity, but do not exclude the emergence of electrostatic charges and require the additional costs for their purchase. The use of INSET allows the removal of charges of static electricity from the objects of their formation into the earth, but not to use this source of energy for certain purposes.

\section{Research methods}

The value of the charge acquired by the liquid during its motion through the pipes is determined with the known 'capillary method' (Rogers and Shlekser, 1961), the essence of which is to determine the value of charge, acquired by a single volume of liquid flowing through a capillary. The investigated liquid from reservoir 1 (Figure 2) runs into the insulated reservoir 2 . The charge formed in the liquid flowing through the capillary 3 , being accumulated in the reservoir 2, changes its potential. According to the measured value of the potential and the known capacity of the system, the value of the charge acquired by the liquid is determined from the formula:

$$
Q=C U
$$

where 
$Q \quad$ is the value of the charge, acquired by the liquid, C

$C$ electric capacity, $\mathrm{F}$

$U$ the value of the potential (voltage), $\mathrm{W}$.

The magnitude of the charge and the known volume of fluid that has already overflowed are determined based on the average charge density. Variable capacitor 5 works to compensate the system volume changes. The potential value is measured with the electrostatic voltmeter 4 .

Figure 2 Principal scheme of the determination of electrification by capillary method

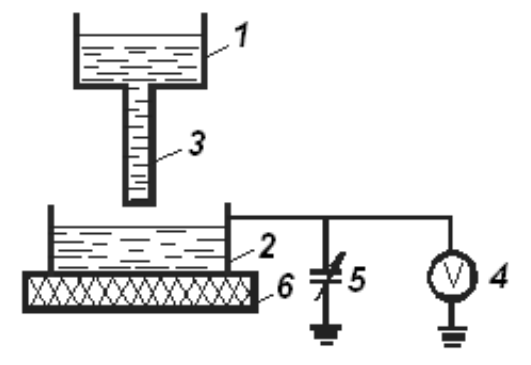

Notes: 1 - tank (tank); 2 - tank (tank) with electrical insulation; 3 - capillary;

4 - electrostatic voltmeter; 5 - alternating capacitor; 6 - electric insulator.

The charge value in the filter and after the filter was determined with the well-known method 'Esso' by the company 'Shell' (Rogers and Mundoy, 1980). The schematic diagram of the 'ER' device used by 'Esso' company to assess fluid electrification is given in Figure 3

Figure 3 Schematic diagram of the device 'ER' for the evaluation of fuels electrification

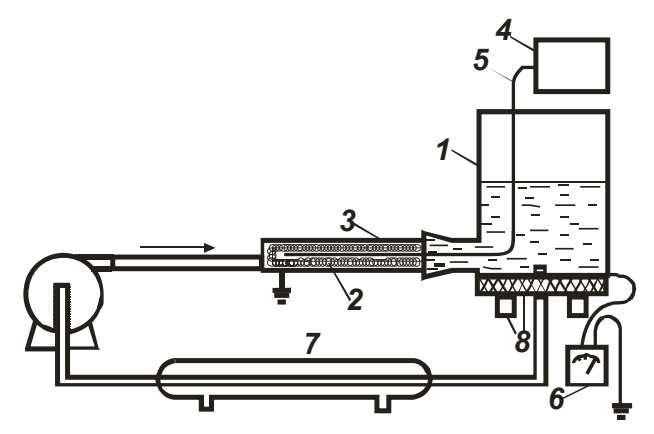

Notes: 1 - tank (tank) with electrical insulation; 2 - metal pipe; 3 - filter element; 4 - radio; 5 - antenna; 6 - ammeter; 7 - fridge.

The insulated reservoir 1 is filled with 3.75 litres of liquid that is continuously pumped through a stainless steel pipe $225 \mathrm{~mm}$ in diameter filled with glass fibre, at the rate of $15.2 \mathrm{l} / \mathrm{min}$. The magnitude of the charge generated in the fuel during pumping is estimated by the number of discharges in the filter 3, recorded by the receiver 4 with the antenna 5, and the leakage current from the reservoir to the ground are measured with the 
micro-ammeter 6 after ten minutes of pumping. The refrigerator 7 serves to stabilise the temperature of the liquid.

To conduct a full-scale experiment on the study of the electrification of hydrocarbon fluids, an appropriate stand was developed. The basic scheme of the stand is depicted at Figure 4

Figure 4 Principal scheme of the test stand

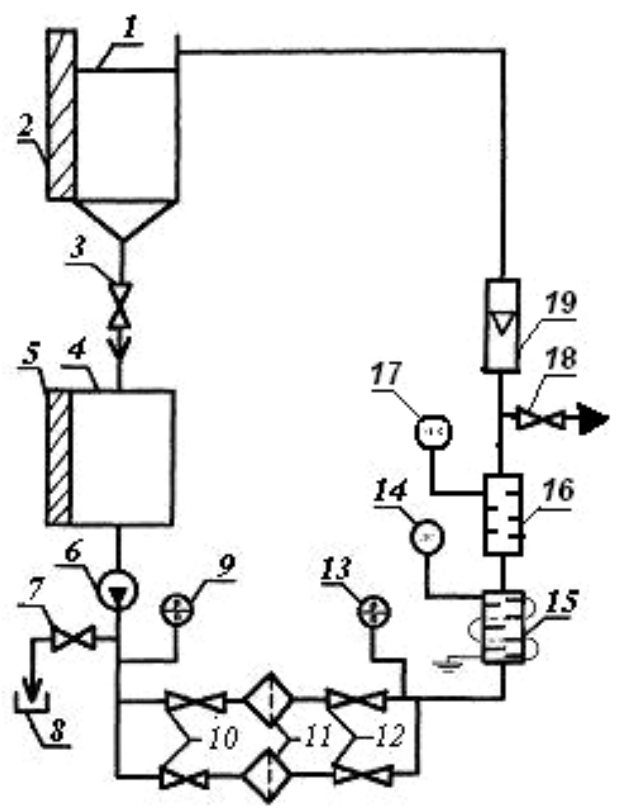

Notes: 1, 4-tanks; 2, 5- measuring glass; 3, 7, 10, 12 - cranes; 6 - pump for pumping liquids; 9, 13 - pressure gauges; 11 - filters (with different filter material); 14 - current source; 15 - device for generation of high voltage current (development of authors); 16 - industrial electrostatic sensor; 17 - electrometer amplifier 'U1-6'; 18 - sampling crane; 19 - rotameter.

The investigated liquid (JET A-1) from the tank 4 is sent through the pipeline with the help of the centrifugal pump 6 to the filters 11 (one of the filters has metal filter net, the other is a standard filter paper) and, according to the electrification theory, the fluid in the filters receives the maximum level of natural electrification. Further, along the pipeline, the fluid under study enters the device for producing high-voltage electrical energy 15 [development of authors (Zubchenko et al., 2006)], which transfer acquired electrostatic charges to the metal hollow ball. Then the liquid enters the tank 1, from which JET A-1 samples can be taken to evaluate their performance, or they can be taken from the tap 18 . To account the liquid flow, a rotameter 19 is installed into the stand. With the help of pressure gauges 9,13 the necessary pressure is created in the system. Indicators of pressure gauges also show the efficiency of the filters, or the violation of their work. Overlapping cranes 10,12 can be replaced with filter materials, since the filter casing is made easy to assemble. To achieve the greatest effect, it is recommended to open the tap 3 and allow some fluid to get into the circle to reduce the charge relaxation event. To investigate the electrification of hydrocarbon media, the device 15 should be switched off 
(in this case it is used as a source of natural electrification) and the electrometric amplifier 17 (type 'U1-6') should be switched on, which will not only amplify the electrical signal received from the electrification sensor 16 , but it will also enable a wide range of measurements with the built-in micro-ammeter and voltmeter.

The charge in the tank is recorded with electrostatic voltmeter. The conductivity is measured with the electrometer amplifier 'U1-6' with the adjustable current source. The dielectric constant is determined with the AC bridge 19 (P-570). Determination of resistance and dielectric permittivity has been conducted discretely by sampling. Characteristics of the metering accuracy for all the applied devices and parameters are within the acceptable limits (Table 1).

Table 1 Characteristics of the metering accuracy

\begin{tabular}{llcc}
\hline$N$ & \multicolumn{1}{c}{ Defined parameter } & Method of determination & Maximum error \\
\hline 1 & Electric capacity & Measurement & $0.1 \%$ \\
2 & Dielectric permeability & Indirect & $\pm 0.2 \%$ \\
3 & Potentials difference & Measurement & $\pm 5.0 \%$ \\
5 & Specific volumetric density of & Indirect & $\pm 6 \%$ \\
& electrostatic charge & & $\pm(5 \%-20 \%)$ \\
6 & Resistance to charge with liquid & Measurement & $\pm(5 \%-20 \%)$ \\
7 & Resistance & Indirect &
\end{tabular}

In this fuel scheme, there are several mechanisms for the formation of electric charge: in the flow through the pipeline, at the filtration and due to fuel spraying in the tank. In this regard, the measurement scheme involves controlling the charge generated in three sections of the stand: in the pipeline, on the filter and in the tank. The charge density generated in the pipeline is related to the current of the outflow from the walls of the pipe with the ratio:

$$
q_{t}=I_{t} / V_{m}
$$

where

$q_{t} \quad$ volume density of charge, $\mathrm{C}$

$I_{t} \quad$ outflow current from the walls of the pipe, A

$V_{m}$ bulk fuel flow, $\mathrm{m}^{3} / \mathrm{sec}$.

Measuring $V_{t}$ and $I_{t}$ you can determine the average $q_{t}$. The structure of the sensor 16 (see Figure 4) represents a pipe section with a diameter equal to the diameter of the pipelines. To remove obstacles, the sensor is placed on the screen. Similarly, as described above, the charge density generated in the filter is characterised with the amount of outflow current from the filter and the volume of fuel flow through the filter according to the equation:

$$
q_{f}=I_{f}\left(1-e^{-t / \tau}\right) / V_{f}
$$

where

$I_{f} \quad$ outflow current from the filter, A 
$V_{f}$ volume fuel flow (in our case it is equal to the flow through the pipeline), $\mathrm{m}^{3} / \mathrm{sec}$

$q_{f}$ volume density of the charge formed in the filter, $\mathrm{C} / \mathrm{m}^{3}$

$\tau \quad$ fluid flow passage time along the sensor, sec

$t$ time of liquid staying in the filter, sec.

Equation (3) takes into account the charge relaxation in the filter through the component $e^{-t / \tau}$. The estimate of the time $t$ and $\tau$ showed that they are about 0.08 seconds and 1.5 seconds in accordance. And this means that this component of equation (3) $\left(e^{-t / \tau}\right)$ can be taken as zero. Experimental verification to register the current flow in the pipe after the filter has showed the correctness of the above assessment. The outflow current from the pipeline if the relaxation in the filter (the filter is grounded) is provided and if it is absent (the filter is electrically isolated from the system) was the same. Accordingly, it can be concluded that the grounding of the filter does not have a significant effect on the formation and distribution of the charge in the system. This has been become the basis for using the filter as a sensor, provided that it is electrically insulated from the structure of the stand with fluoroplastic insulators.

It is known that the charge is acquired by fuel at the filter and along the pipeline, transporting it into the tank. On this way, it is scattered in the pipeline. Therefore, the amount of charge that has fallen into the tank is less than that formed at the filter and the pipeline. However, charge formation in the tank itself may occur due to spraying, mixing, bubbling, deposition, etc. To evaluate the charge in the fuel tank, an electrode isolated from the tank wall has been introduced in the middle of the tank. The difference in the potentials on the plates of such capacitor depends on the charge in the tank according to the formula:

$$
Q_{b}=C_{o} U_{e-t} k(h)
$$

where

$Q_{b} \quad$ is the total charge in the tank, C

$C_{o} \quad$ electric capacity at the measured vessel: electrode-tank under the absence of fuel, $\mathrm{F}$

$U_{e-t}$ potential difference: measuring electrode-tank, $\mathrm{W}$

$k(h)$ index, accounting the measurement of the system capacity, depending on the fuel level.

The experimental determination of the index $k(h)$ based on the capacitance measurements as a function of the fuel level has showed that its value varied from 1 (at empty tank) to 0.99 (when the tank is full). Measurements of resistance and dielectric permittivity have also been performed to determine the relaxation time and control of the results repeatability.

The study was carried out with the fuel TS- 1 at the following fixed values: temperature $\mathrm{T}=20^{\circ} \mathrm{C}, \rho=4.10^{11} \Omega \cdot \mathrm{m}, \varepsilon=2.06, \tau=7 \mathrm{sec}, \xi=1.3 \mathrm{cSt}$. The dependence of the leakage current from the sensor wall on the average flow rate is given in Figure 5 (curve 2) and is a power function with an index of degree 0.5-0.7. To facilitate comparison, all functions in Figure 5 are represented in relative units, for which the maximal experimental values of the investigated quantities are selected. The deviation from the known theories, which establish the power factor of 1.5-2 for this dependence, 
is explained by the fact that at limited length of the sensor the ratio of the time of fuel staying at the sensor and the time of the relaxation of fuel affects the process strongly. The time of fuel staying at the sensor in this case is shorter than the time for the formation of the double layer, so the charge density falls. When the liquid flows through the pipeline with the internal diameter of $12 \mathrm{~mm}$ at the rate of $0.7 \mathrm{~m}^{3} /$ hour, fuel is retained in the sensor of $320 \mathrm{~mm}$ long for 1 second. The time of the double layer formation is determined by the relaxation time and it is 1.6-1.7 sec for the JET A-1 fuel with the specific resistance of, $10^{11} \Omega$ and the relative permittivity $\varepsilon=2$. With the known dependence that takes into account the ratio $t / \tau$, it is possible to eliminate the influence of the length of the sensor measurement results by determining $I_{t o}$ - current flow for infinitely long sensor (curve 3 at Figure 5).

Figure 5 The dependence of JET A-1 fuel electrification on the (1) flow rate in the filter, (2) in the pipes before the filter, (3) in the filter from the pipes walls and (4) after the filter

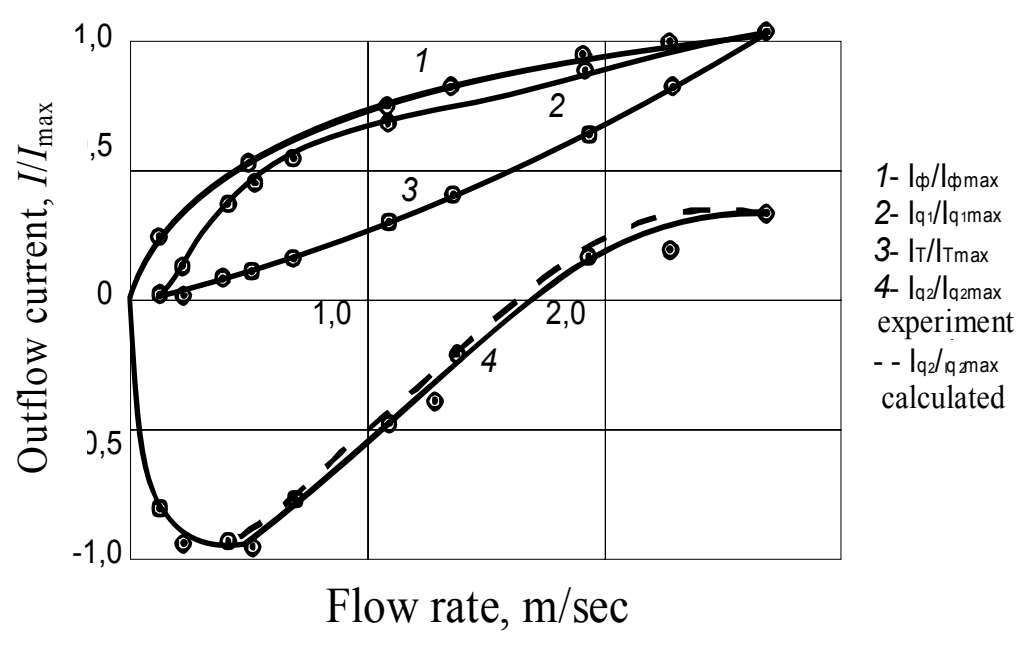

The obtained dependence is consistent with the known research results (Scanavi, 1991; Rogers and Mundoy, 1980; Taubkin, 2018). The density of the charge formed by the flow in the pipe is determined by the dependence (2). In order to compare the calculated results with the experimental ones, the rate gradients at the border of the viscous layer and the turbulent core were determined. The border is the lower boundary of the logarithmic stratum.

The definition of $\Delta$ has been carried out as follows. Using the known dependence $\Delta / \operatorname{Re}=f(\operatorname{Re})$ for smooth tubes (Chebotarev, 2003) the ratio $\Delta / R$ has been defined for the test mode currents, set in the pipe, and then $\Delta$ has been derived from the known $R$. The value grad $v(r)$ has been determined by the differentiation $v=f(r)$ :

$$
\operatorname{grad} v(r)=\frac{4 v_{\text {cep }}(R-\Delta)}{R^{2}}
$$

where

$v \quad$ average flow rate of the liquid, $\mathrm{m} / \mathrm{sec}$

$R \quad$ radius of the pipe, $\mathrm{m}$ 
$\operatorname{grad} v$ fluid flow rate gradient, $\mathrm{m} / \mathrm{sec} \cdot \mathrm{mm}$

Re Reynolds criterion

$\Delta \quad$ thickness of the viscous substrate, $\mathrm{m}$.

The dependence of the density of the charge generated in the pipeline on the gradient of the TC-1 fuel flow rate at the boundary of the turbulent core is shown at Figure 6

Figure 6 Dependence of the charge density in the pipe on the flow rate gradient of the aviation fuel of JET A-1

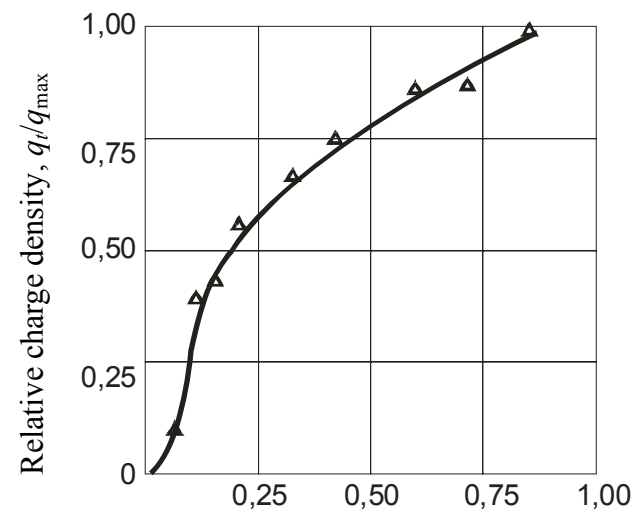

Rate gradient, $\operatorname{grad} v, \mathrm{~m} / \mathrm{sec}^{*} \mathrm{~mm}$

The character of the obtained dependence is similar to the dependence of the fuel charge density on the flow rate gradient obtained in laboratory studies. This proves the similarity of the processes taking place in the pipe and the measuring cell and confirms the correctness of the conclusion about the decisive influence of the flow rate gradient on the process of charges separation.

The comparison of the experimental dependences $I_{t} / I_{\text {max }}$ (Figure 5) and $q_{t} / q_{t \max }$ (Figure 6) shows that at a shorter length of the sensor, the sensor current is much less proportional to the fuel charge density, gained while flowing through the long pipeline of the same diameter. In fact, already at $l=0,1 v \tau$ we obtain the following dependence:

$$
1-e^{-\frac{l}{v \tau}} \approx \frac{l}{v \tau}
$$

where

$l \quad$ is the length of the sensor, $\mathrm{m}$

$\checkmark$ flow rate, $\mathrm{m} / \mathrm{sec}$

$\tau$ fluid flow passage time along the sensor, sec.

Now the equation for finding the charges formation current in the pipe takes the form:

$$
I_{t}=k q
$$

where $q$ is the charge density, measured inside the pipe, $\mathrm{C} / \mathrm{m}^{3}$. 
Figure 5 (curve 4) shows the change in the sensor current set after the filter, depending on the flow rate. Comparison with the sensor current to the filter (curve 2) shows that at low fuel flow, the outflow current dominates, resulting in the change in the direction of total current. As the flow increases, the outflow current decreases and at high flow, the current becomes lower, than that ensured by charging fuel in the sensor. The total current receives the same sign as the current from the sensor to the filter, but its absolute value is lower. The analytical conclusion leads to the following equation:

$$
I_{g_{2}}=I_{t}\left(1-e^{-\frac{l_{d t .}}{v \tau}}\right)-I_{f} e^{-\frac{l_{d t . f}}{v \tau}}\left(1-e^{-\frac{l_{d t .}}{v \tau}}\right)
$$

where

$l_{d t . f}$ distance from the filter to the sensor

$l_{d t}$ length of the sensor.

The current $I_{g_{2}}$ determined from the formula (8) based on the measured values of $I_{t}$ and $I_{f}$ as a function of flow is given in Figure 5 (intermitted lines). Thus, we can state that in the pipe of small length the current determined from the formula (8) is equal to the flow current only in the case when uncharged fuel enters this pipe. When the charged fuel gets into the pipe, the current is more complex function of the current flow. Moreover, with increasing flow rate, the current from the pipe decreases, despite increasing the flow current. It is obvious that the usual assessment of the degree of electrification by the flow current in the latter case is wrong. The nature of the processes occurring in the filter is likely to be similar to the phenomena in the pipes. This conclusion can be drawn from the consideration of the dependence of the charge density in the filter on the flow rate of the aviation fuel of JET A-1 brand (Figure 7).

Figure 7 Dependence of the charge density generated in the pipe and the filter on the flow rate of the aviation fuel of JET A-1

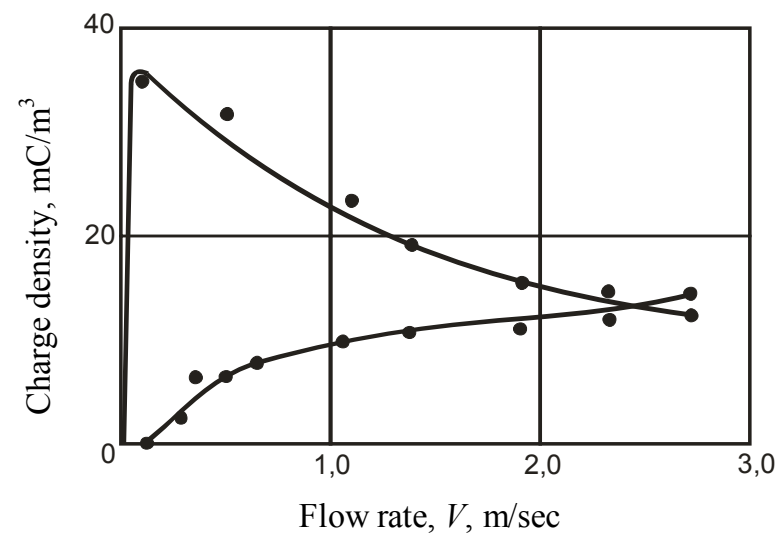

The highest charge density, formed in the filter, is observed at low flow. As the rate increases, the charge density $q_{f}$ decreases and tends to reach certain constant value at high rates. This can be explained by the same reasons as for the flow in the pipe. At low flow rate, the charge increases in proportion to the rate gradient. When the value $v \tau$ becomes 
larger than the thickness of the filter element, the double layer, destroyed by the forces of the hydrodynamic field, does not have time to recover and the charge density decreases. At infinite pipe length, the charge density approximates the certain constant value and does not depend on the place of formation - in a filter or in a pipe.

As a result of theoretical and experimental research, a device and method for generating high-voltage electric power (Zubchenko et al., 2006; Trofimov et al., 2006) has been developed and tested. It is based on the positive use of static electricity charges obtained with the help of INCET for the operation of hydrocarbon fuels.

Figure 8 shows the schematic diagram of the device for receiving high voltage power.

Figure 8 Device for receiving high voltages power

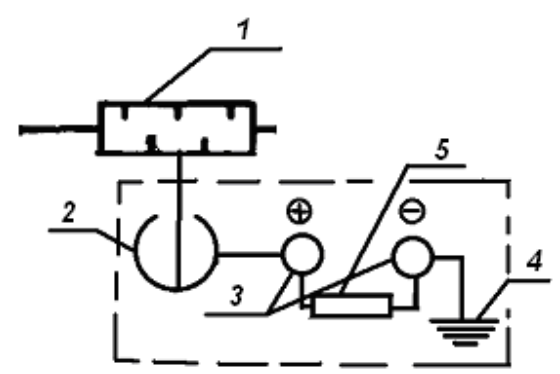

Notes: 1 - INSET, 2 - hollow metal ball, 3 - arrester, 4 - ground, 5 - load.

The device works as follows: charges of static electricity are constantly accumulated in the elements of technological equipment and with the help of INSET 1 they are taken to the metal ball 2, and then to the arrester 3 , one end of which is connected to the bullet 2 , and the second is connected with the ground 4 . The arrester 3 is connected to the load 5, the voltage on which leads to the appearance of electric current in the electrical circuit. By changing the gap size of the electric discharger it is possible to obtain the required value of the potential on a metal ball 2. For the case of the dangerous potential level formation, the air discharge is provided for which the discharger 3 is grounded.

From the technological equipment (see Figure 4) static electricity charges are removed by the INSET 16 . But unlike its standard application, when the collected static electricity charges are discharged into the ground, in this method, static electricity charges from INSET 16 are diverted to high voltage electrical energy by the device (Figure 8), which allows receiving current of 20-30 kV.

\section{Conclusions and discussion}

All the investigated types of dielectric liquids are significantly electrified. At turbulent flow in pipes the flow current is proportional to the flow rate raised to the power of 1.75-2. In case of the laminar flow, it linearly depends on the flow rate of the liquid, as it is shown by the data from the filters. The absolute value of the charge, which is obtained by the fuel, depends on the properties of the liquid itself: purity, composition, etc. and on 
the characteristics of the flow, structural features of the equipment and external influences. However, the question of the conditions, necessary for the formation and accumulation of charges, is still insufficiently studied.

Our studies of the electrification of dielectric liquids, flowing in pipes and during filtration, have output the following main results:

- The influence of the filling rate on the amount of excess charge in the tank has been confirmed.

- The dependence of JET A-1 fuel electrification on the flow rate in the filter and pipes to the filter and after the filter has been confirmed.

- The dependence of the charge density, arising in the pipe and the filter, on the flow rate and the rate gradient has been established. The nature of the obtained dependence is similar to the dependence of the fuel charge density on the flow rate gradient, obtained in laboratory studies. This indicates the similarity of the processes occurring in the pipe and the measuring cell and confirms the correctness of the conclusion about the decisive influence of the flow rate gradient on the process of the charges separation.

- It has been established that the outlet current from the pipeline is the same both when the relaxation in the filter is present (the filter is grounded) and when it is absent (the filter is electrically isolated from the system). Accordingly, it has been established that the grounding of the filter does not affect significantly the formation and distribution of charge in the system.

- The experimental determination of the index $k(h)$ as a function of the fuel level has shown that its value has changed from 1 (at empty tank) to 0.99 (at the full tank).

- Comparison of the experimental dependences of $I / I_{\max }$ and $q_{t} / q_{\max }$ shows that at a shorter length of the sensor, the sensor current is much lower than the density of the charge taken up by the fuel when flowing in a long pipeline of the same diameter.

- It has been established that as the flow rate increases, the outflow current decreases and at a high flow it becomes lower than the current, provided by charging fuel in the sensor. The total current receives the same sign as the current from the sensor to the filter, but its absolute value is lower.

- It is established that in a short pipe the current is equal to the flow current only when it enters the uncharged fuel. When the charged fuel gets into the pipe, its current is more complex function of the current flow. Moreover, with increasing flow rate, the current from the pipe decreases, despite the increase in the current flow.

- It has been established that the highest charge density, formed in the filter, is observed at low flow. As the speed increases, the charge density decreases and reaches a constant value at high rate.

- The new device and method for generation of high voltage power from the distribution of charges in the dielectric liquids have been developed. 


\section{References}

Chebotarev, L.I. (2003) Operation of Airports Fuel Supply Equipment, Air. transport., Moscow.

Ilin, A.A. and Dzhioeva, T.B. (2018) 'Analysis of material properties for fabrication of pipelines aimed at reducing accumulation of static electricity in the process of hydrocarbons pumping', Transactions of the Krylov State Research Centre, No. 1, pp.55-61, DOI: 10.24937/25422324-2018-1-S-I-55-61.

Pinchuk, V.A. (1997) 'Motor electrification as a phenomenon, reflecting the development of charge instability in the environment of the combustion products upon expiration', Journal of Technical Physics, Vol. 67, No. 8, pp.21-26.

Pribylov, V.N. (2003) 'Formation of an Electric Charge in a Dielectric Fluid Above a Rotating Disk', Colloid Journal, Vol. 65, No. 4, pp.488-492 [online] https://doi.org/10.1023 /A:1025181104973.

Rogers, D.T. and Mundoy, I.C. (1980) Products Research Division, Report N RL-YM-60, Esso Resarch and Engineering Company.

Rogers, D.T. and Shlekser, T.Y. (1961) 'Theoretical and experimental studies of fuel electrification', V International Petroleum Congress, Moscow, Vol. 1. pp.331-335.

Roizen, I.O. and Medvedeva, V.C. (1995) 'Static electricity and measures to combat it in the chemical industry', Protection of Chemical Enterprises from Fires and Explosions, pp.285-92, Research Institute of Technical and Economic Research in the Chemical Complex, Moscow.

Samoilescu, G. (2009) 'Electrostatic field within oil tankers', Rev. Roum. Sci. Techn. Électrotechn. et Énerg., Vol. 54, No. 3, pp.261-270.

Scanavi, G.I. (1991) Dielectric Physics, (Field of Weak Fields), State Publishing House of Technical and Technical Literature, Moscow-Leningrad.

Shell Research Ltd. and Shell Int. Research N.v. (1960) Static Electricity in Aircraft-Faelliny tests on a Simulatca Wing Tank, Thornton Research Centre Rept., M 206, April.

Taubkin, I.S. (2018) 'Overview of static electricity in some industrial operations with petroleum products', Theory and Practice of Forensic Science, Vol. 13, No. 2, pp.54-64, DOI: 10.30764/ 1819-2785-2018-13-2-54-64.

Trofimov, I.L., Zubchenko, O.M. and Kravets, I.A. (2006) Method of Obtaining Electric Energy of High Voltages, Patent, 18479, H02N 1/00, Ukraine.

Zubchenko, O.M., Trofimov, I.L. and Kravets, I.A. (2006) Device for Obtaining High Voltage Electrical, Patent, 13487U, H02N 1/00, H02H 1/06, Ukraine.

\section{Nomenclature}

$v \quad$ average flow rate of the liquid, $\mathrm{m} / \mathrm{sec}$

$\tau \quad$ fluid flow passage time along the sensor, sec

$\Delta \quad$ thickness of the viscous substrate, $m$

$v \quad$ flow rate, $\mathrm{m} / \mathrm{sec}$

C electric capacity, $\mathrm{F}$

Co electric capacity at the measured vessel: electrode-tank under the absence of fuel, $\mathrm{F}$

$\operatorname{grad} v$ fluid flow rate gradient, $\mathrm{m} / \mathrm{sec} \cdot \mathrm{mm}$

$I_{t} \quad$ outflow current from the walls of the pipe, A 
$I_{f} \quad$ outflow current from the filter, A

$k(h) \quad$ index, accounting the measurement of the system capacity, depending on the fuel level

$l \quad$ length of the sensor, $\mathrm{m}$

$l_{d t} \quad$ length of the sensor

$l_{d t . f} \quad$ distance from the filter to the sensor

$q$ charge density, measured inside the pipe, $\mathrm{C} / \mathrm{m}^{3}$

$Q \quad$ value of the charge, acquired by the liquid, C

$q_{t} \quad$ volume density of charge, $\mathrm{C}$

$Q_{b} \quad$ total charge in the tank, C

$q_{f} \quad$ volume density of the charge formed in the filter, $\mathrm{C} / \mathrm{m}^{3}$

$R \quad$ radius of the pipe, $\mathrm{m}$

Re Reynolds criterion

$t$ time of liquid staying in the filter, sec

$U$ potential difference: measuring electrode - tank, $\mathrm{W}$

$U_{e-t} \quad$ the value of the potential (voltage), $\mathrm{W}$

$V_{f} \quad$ volume fuel flow (in our case it is equal to the flow through the pipeline), $\mathrm{m}^{3} / \mathrm{sec}$

$V_{m} \quad$ bulk fuel flow, $\mathrm{m}^{3} / \mathrm{sec}$

$\tau \quad$ fluid flow passage time along the sensor, sec 